\title{
A Rare Instance of Temporomandibular Joint Dislocation Complicated by Motor-Evoked Potential Monitoring
}

\author{
Manjunath Munireddy ${ }^{1}$ Dheeraj Masapu ${ }^{1}$ \\ Ramachandran Govindasamy ${ }^{1}$ \\ 1Department of Neurosciences, Varthur Hobli, Sakra World \\ Hospital, Bellandur, Devarabisanahalli, Bengaluru, Karnataka, India
}

Address for correspondence Dheeraj Masapu, MD, DM, Department of Neurosciences, Devarabisanahalli, Varthur Hobli, Sakra World Hospital, Bellandur, Bengaluru 560103, Karnataka, India (e-mail: dheerajmasapu@gmail.com).

limbs, tibialis anterior and adductor hallucis longus in the lower limbs. The impedance of all electrodes was maintained below $5 \mathrm{kV}$. The stimulus strength was determined by giving currents starting from $150 \mathrm{~V}$ and gradually escalating up to a strength of $300 \mathrm{~V}$ until good compound motor action potentials (CAMPs) were elicited. The depth of anesthesia was maintained within a bispectral index range of 30 to 40 . AAD was reduced by gentle manipulation with the head fixed on Sugita frame. Intraoperatively, C1 to C2 fusion was performed with Magerl transarticular screw and the onlay bone grafting was obtained from iliac autograft. Following surgery, the patient was extubated uneventfully.

In the immediate postoperative period, patient developed jaw tightness and was unable to close his mouth completely. On examination (-Fig. 1A), he had fullness in both sides of the face, jaw trismus with a gap in the inter incisor area. On palpation of the cheek, there was a sizeable gap of one fingerbreadth in the TMJ indicating dislocation. The same was confirmed using a computed tomography (CT) of the face and neck showing grade 2 joint dislocation ( - Fig. 1B). ${ }^{3}$ An urgent plastic surgeon opinion was sought and the TMJ was reduced via an intraoral closed reduction technique ( - Fig. 1C) under moderate sedation. ${ }^{4}$

The patient made a good recovery with no complications or focal neurological deficits. CT scan was done to assess the position of the screw in $\mathrm{C} 1$ to $\mathrm{C} 2$, which revealed an improper screw position. It was decided to go for a revision surgery of C1 to C2 fusion with an iliac bone graft. During the revision surgery, we intubated him via nasal route using fiberoptic bronchoscope and kept a stabilization brace, which wraps the face ( - Fig. 1D) and forces the mandible upward, preventing potential dislocation. The patient was then placed in the prone position and transcranial MEPs were recorded. We removed malpositioned transarticular screws and modified it using C1 to C2 Goel's fusion with C1 lateral mass, C2 pars screws, and rods. A C-arm "X-ray check" was performed on table at the end of the surgery before extubation. It showed intact TMJ and the chin strap was kept in place for few more hours until the patient was fully awake.
DOI https://doi.org/ $10.1055 / \mathrm{s}-0039-1685417$ ISSN 2348-0548.
Copyright $\odot 2020$ Indian Society of Neuroanaesthesiology and Critical Care
License terms

() (1) $\Theta \circledast$ 


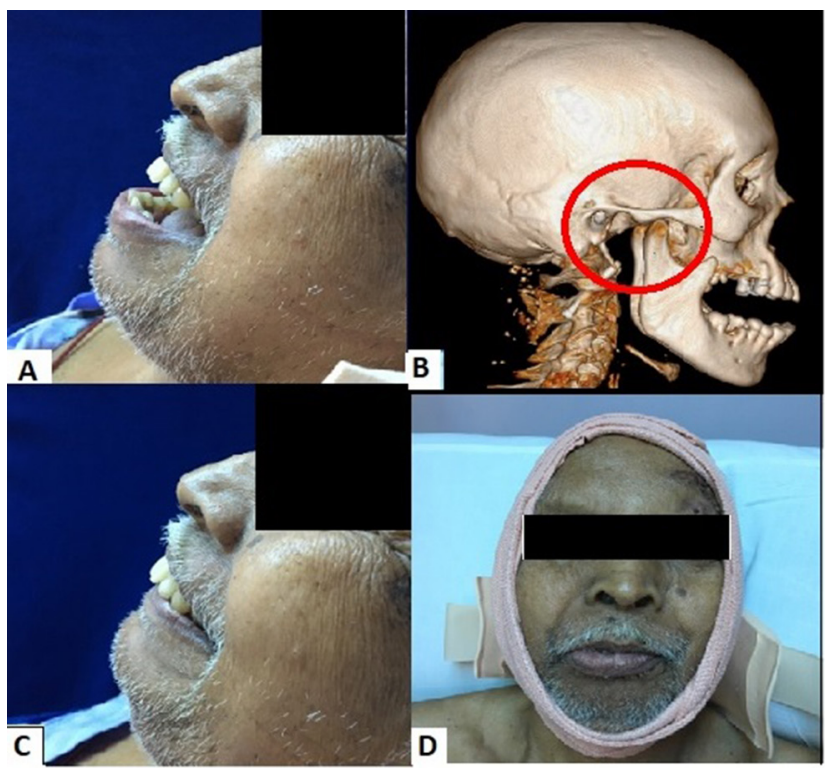

Fig. 1 (A) Image showing the inability to close the oral cavity (open lock). (B) CT 3D model showing the dislocated temporomandibular joint (in the circle). 3D, three-dimensional; CT, computed tomography. (C) After intraoral closed reduction technique. (D) Chin strap to prevent dislocation.

Temporomandibular joint dislocation is associated with severe pain and tenderness over the jaw. If left untreated for more than 14 days, it may lead to fibrosis of the dislocated joint and rare cases of TMJ fracture have been reported. These complications may require surgical treatment. ${ }^{5}$ To the best of our knowledge, TMJ dislocation during MEP recording has not been reported in the literature till date. We hypothesize that in our case, MEP recording would have stimulated the facial motor cortex, contributing to the activation of facial muscles leading to TMJ dislocation. The main priority during intubation for anesthesia is to prevent any maneuvers leading to aggressive movement at the TMJ, thereby dislocating it. Hence, during the revision surgery, we made modifications such as nasotracheal intubation, chin strap ( - Fig. 1D), and low voltage stimulation to elicit MEPs. Techniques requiring low voltage stimulation for acquiring better MEPs include multitrain electrical stimulation, ${ }^{6}$ D-wave monitoring, ${ }^{7}$ and double train transcranial electrical stimulation. ${ }^{8}$ It also warrants adequate attention for identification of at-risk individuals during preoperative assessment and instituting the above-mentioned principles for prevention and early management of dislocation.

In conclusion, TMJ dislocation is one of the underrated potential complications that has to be carefully considered during MEP monitoring for any spinal surgery. Preanesthetic evaluation of at-risk individuals needs adequate precautions and modifications in the surgical and anesthesia management techniques that might limit joint mobility and prevent TMJ dislocations.

\section{Conflict of Interest}

None declared.

\section{References}

1 Awsare AN, Prakash N. Temporomandibular dislocation: should every doctor be trained in resetting the jaw? Br J Oral Maxillofac Surg 2006;44(4):339

2 Kuwahara Y, Taguchi S, Kuroda M, Kawamoto M. Adverse events during transcranial muscle evoked potential monitoring [article in Japanese]. Masui 2011;60(6):692-696

3 Akinbami BO. Evaluation of the mechanism and principles of management of temporomandibular joint dislocation. Systematic review of literature and a proposed new classification of temporomandibular joint dislocation. Head Face Med 2011;7(1):10

4 Pillai S, Konia MR. Unrecognized bilateral temporomandibular joint dislocation after general anesthesia with a delay in diagnosis and management: a case report. J Med Case Reports 2013;7(1):243

5 Han I, Kim TK, Yoo JH, Park JH, Chung EY. Dislocation of the temporomandibular joint following general anesthesia. Korean J Anesthesiol 2014;67(Suppl):S113-S114

6 Ushio S, Kawabata S, Sumiya S, et al. A multi-train electrical stimulation protocol facilitates transcranial electrical motor evoked potentials and increases induction rate and reproducibility even in patients with preoperative neurological deficits. J Clin Monit Comput 2018;32(3):549-558

7 Costa P, Peretta P, Faccani G. Relevance of intraoperative D wave in spine and spinal cord surgeries. Eur Spine J 2013; 22(4):840-848

8 Journée HL, Polak HE, de Kleuver M, Langeloo DD, Postma AA. Improved neuromonitoring during spinal surgery using double-train transcranial electrical stimulation. Med Biol Eng Comput 2004;42(1):110-113 